\title{
Precise positioning of spacecrafts by multi-frequency VLBI
}

\author{
Yusuke Kono ${ }^{1}$, Hideo Hanada ${ }^{1}$, Ping Jinsong ${ }^{2}$, Yasuhiro Koyama ${ }^{3}$, Yoshihiro Fukuzaki ${ }^{4}$, and Nobuyuki Kawano ${ }^{2}$ \\ ${ }^{1}$ Division of Earth Rotation, National Astronomical Observatory, 2-21-1 Osawa, Mitaka, Tokyo 181-8588, Japan \\ ${ }^{2}$ Division of Earth Rotation, National Astronomical Observatory, 2-12 Hoshigaoka, Mizusawa, Iwate 023-0861, Japan \\ ${ }^{3}$ Kashima Space Research Center, Communications Research Laboratory, 893-1 Hirai, Kashima, Ibaraki 314-0012, Japan \\ ${ }^{4}$ Geographical Survey Institute, 1 Hokugou, Tsukuba, Ibaragi 305-0811, Japan
}

(Received July 17, 2002; Revised October 15, 2003; Accepted October 21, 2003)

\begin{abstract}
Multi-Frequency VLBI (MFV) is one of the most powerful methods for precise positioning of spacecrafts. The system transmits three carrier waves at S-band and one wave at X-band. These frequencies are set to resolve the cycle ambiguity of carrier wave at X-band from two group delays between carrier waves and one phase delay of the carrier wave at S-band. The procedure to resolve the cycle ambiguity is proposed in this article. Some conditions about frequency variation and prediction of position and the ionosphere are also clarified for resolving the cycle ambiguity. The dedicated recording system for MFV is developed. A preliminary observation of MFV is carried out with this system by using Lunar Prospector. As a result of the experiment, residual phases from predicted ones are within $\pm 2 \pi$, and the RMS of the residual for the period of several seconds is about 4 degrees. This result supports realization of the MFV.
\end{abstract}

Key words: VLBI, positioning, spacecraft, SELENE.

\section{Introduction}

The study of orbital motion and the interior structure of the Moon and planets is one of the methods to learn about solar system revolution as a dynamical system and the origin of the solar system. Measuring the gravity fields obtained by the orbital motion of a spacecraft is a powerful method to estimate the inner layer and the density structure of the Moon and the planets. Orbits or positions of a lunar or planetary spacecraft have mainly been determined by range and Doppler measurements. These measurements provide only one-dimensional information about the position along the line of sight. On the other hand, differential VLBI (Very Long Baseline Interferometry) has the sensitivity of positioning in directions perpendicular to the line of sight, so that combining both range and Doppler measurements with differential VLBI enables us to measure three-dimensional position of a spacecraft. VLBI methods have been used for positioning of spacecrafts since the 1960's. In these methods, carrier waves were transmitted from a spacecraft in order to save its transmitting power and obtain high SNR. Unfortunately, the phase delay of a carrier wave, however, has cycle ambiguities, therefore phase delay rate and group delay have been mainly used so far (e.g., Sagdeyev et al., 1992; Border et al., 1992).

A new VLBI method, "Multi-Frequency VLBI (MFV)" is proposed. Two spacecrafts transmit three frequency signals in S-band and one signal in X-band. Ground VLBI stations receive the signals by beam switching method in the case of the larger angular distance between the radio sources than the beamwidth of the antennas or by same beam method in

Copy right(c) The Society of Geomagnetism and Earth, Planetary and Space Sciences (SGEPSS); The Seismological Society of Japan; The Volcanological Society of Japan; The Geodetic Society of Japan; The Japanese Society for Planetary Sciences. the case of the smaller angular distance between the radio sources. These frequencies are set to resolve the cycle ambiguity of a carrier wave at X-band from three group delays between carrier waves at S-band. MFV method which uses phase delay contributes precise positioning of the spacecrafts and gravity field recoveries of the Moon and planets. A procedure to resolve the cycle ambiguity is proposed in this article. Some conditions about frequency variation and prediction of position and the ionosphere are also clarified for resolving the cycle ambiguity. The dedicated recording system for MFV is developed. A preliminary observation of MFV is carried out with this system by using Lunar Prospector. With this result, possibility of realization of MFV is shown in this article.

\section{MFV Method}

In this section, the procedure to resolve the cycle ambiguity of carrier waves and to obtain the precise phase delay is shown. Some conditions for realization of MFV and the accuracy of the geometric delay of MFV are discussed.

\subsection{Cycle ambiguity resolving of carrier waves}

In MFV method, two radio sources respectively emit three carrier waves $s_{1}, s_{2}, s_{3}$ in S-band and one wave $x$ in X-band. The basic concept is shown in Fig. 1. Cross correlation between the four respective carrier waves at two ground stations produces four $\times$ two fringe phases for each unit integration time at first. By differencing the fringe phases between two radio sources, four differenced fringe phase $\phi_{o b s, i}$ are obtained for the carrier wave $i\left(=s_{1}, s_{2}, s_{3}\right.$ and $\left.x\right)$. The phases can be expressed as follows,

$$
\phi_{o b s, i}=2 \pi f_{i} \tau_{r e s, j}+2 \pi N_{i}-2 \pi k D \frac{1}{f_{i}}+\left[\left[\sigma_{\phi_{o b s}}\right]\right],
$$




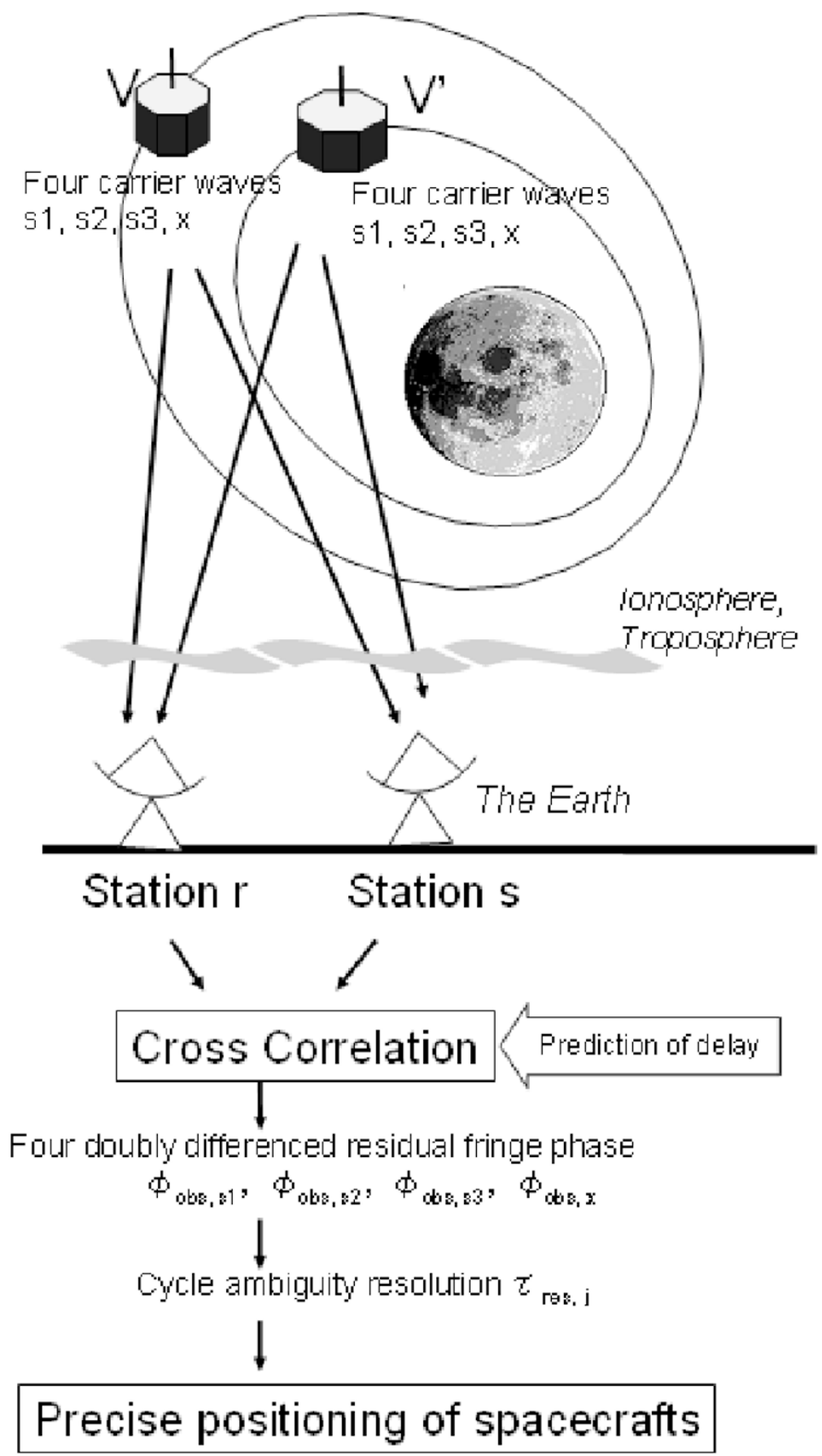

Fig. 1. Basic concept of MFV.

where $f_{i}$ is the frequency of the carrier waves and $\tau_{r e s, j}(j=$ $s, x)$ is the error in the predicted geometric delay differenced between sources. The two radio sources transmit the carrier waves at the same frequencies. $\tau_{r e s, j}$ include tropospheric and instrumental delay differenced between sources. The difference between $\tau_{\text {res,s }}$ and $\tau_{\text {res, } x}$ is mainly originated in the difference between positions of the S-band and X-band antenna. The difference cannnot be known in the cases of satellites which have no attitude sensor. $N_{i}$ is the cycle ambiguity of the phases, $k$ is the constant $\left(k=1.34 \times 10^{-7}\right.$ $\left[\mathrm{m}^{2}\right.$ electrons $\left.\left.{ }^{-1} \mathrm{~s}^{-1}\right]\right), \mathrm{D}$ is the doubly differenced Total Elec- tron Content (TEC) [electrons $\mathrm{m}^{-2}$ ] in the ionosphere along the signal propagation paths and $\left[\left[\sigma_{\phi_{o b s}}\right]\right]$ is the observed phase noise. The parentheses [[ ] ] indicates the error which obeys the error theorem. For example, the relation, $[[a]] \pm$ $[[\mathrm{b}]]=\left[\left[\sqrt{a^{2}+b^{2}}\right]\right]$ stands up.

It is the most advantageous to determine the phase delay of the X-band signal, since the accuracy of the phase delay is proportional to the frequency of the carrier signal on an assumption that the phase error is independent of the frequency. The other three equations are used only for the re- 
solving of the cycle ambiguities of the signals at both $\mathrm{S}$ and $\mathrm{X}$-bands.

In order to obtain the phase delay of the carrier wave in $\mathrm{X}$-band, four procedures are taken as follows,

1) Cycle ambiguity resolution of the wide lane $(s 2-s 1)$

2) Cycle ambiguity resolution of the wide lane $(s 3-s 1)$

3) Cycle ambiguity resolution of the carrier wave $(s 1)$

4) Cycle ambiguity resolution of the carrier wave $(x)$.

The wide lane is one kind of linear combination of two carrier waves (Hatch, 1982). The phase of the wide lane is formed by subtraction of the phase of one carrier wave from that of the other carrier wave. For example, a phase $\phi_{w s 1 s 2}$ of a wide lane is defined as $\phi_{w s 1 s 2}=\phi_{s 2}-\phi_{s 1}$. The frequency of the wide lane corresponds to the frequency difference of the two carrier signals. The name, "wide lane" comes from the fact that the interval between the cycle ambiguities is wide. A wide lane yields a group delay. The basic concept of the four procedures is to resolve the cycle ambiguity in the order of wider intervals.

First of all, the cycle ambiguity of the wide lane of $s 1$ and $s 2$ carrier waves can be solved. The wide lane phase $\phi_{o b s, w s 2 s 1}$ is formed by subtracting the phase $\phi_{o b s, s 1}$ from the phase $\phi_{o b s, s 2}$ as follows,

$$
\begin{aligned}
\phi_{o b s, w s 2 s 1}= & 2 \pi f_{w s 2 s 1} \tau_{r e s, s}+2 \pi\left(N_{s 2}-N_{s 1}\right) \\
& -2 \pi k D\left(\frac{1}{f_{s 2}}-\frac{1}{f_{s 1}}\right)+\left[\left[\sqrt{2} \sigma_{\phi_{o b s}}\right]\right],
\end{aligned}
$$

where $f_{w s 2 s 1}=f_{s 2}-f_{s 1}$. In order to resolve the cycle ambiguity of $N_{s 2}-N_{s 1}$ in Eq. (2), all terms on the right side must be less than $\pm \pi$ at least. The first term on the right side of the equation is composed of the residual delay from the prediction. The condition of $\tau_{\text {res }, s}$ which is the accuracy of the prediction of the geometric delay can be obtained as follows,

$$
\left|\tau_{r e s, s}\right|<\frac{1}{2 f_{w s 2 s 1}} .
$$

The third term on the right side of Eq. (2) is the phase fluctuated by the ionosphere. The condition of $D$ is yielded as well as the first term,

$$
|D|<\left|\frac{f_{s 1} f_{s 2}}{2 k\left(f_{s 1}-f_{s 2}\right)}\right| .
$$

The fourth term on the right side of Eq. (2) is caused by the phase noise of the received signal. The condition is also yielded as follows,

$$
\sigma_{\phi_{o b s}}<\frac{\pi}{\sqrt{2}} .
$$

The validity of these conditions will be discussed in the later section.

Secondly, the cycle ambiguity of the wide lane of $s 1$ and $s 3$ carrier waves will be resolved. The phase $\phi_{o b s, w s 3 s 1}$ of the wide lane is given as follows,

$$
\begin{aligned}
\phi_{o b s, w s 3 s 1}= & 2 \pi f_{w s 3 s 1} \tau_{r e s, s}+2 \pi\left(N_{s 3}-N_{s 1}\right) \\
& -2 \pi k D\left(\frac{1}{f_{s 3}}-\frac{1}{f_{s 1}}\right)+\left[\left[\sqrt{2} \sigma_{\phi_{o b s}}\right]\right],
\end{aligned}
$$

where $\phi_{o b s, w s 3 s 1}=\phi_{o b s, s 3}-\phi_{o b s, s 1}, f_{w s 3 s 1}=f_{s 3}-f_{s 1}$. By eliminating $\tau_{r e s, s}$ from Eqs. (2) and (6), the following equation is obtained.

$$
\begin{aligned}
& \frac{\phi_{o b s, w s 3 s 1}}{2 \pi f_{w s 3 s}}-\frac{\phi_{o b s, w s 2 s 1}}{2 \pi f_{w s 2 s 1}} \\
& =\frac{N_{s 3}-N_{s 1}}{f_{w s 3 s 1}}-\frac{N_{s 2}-N_{s 1}}{f_{w s 2 s 1}}+k D\left(\frac{f_{s 2}-f_{s 3}}{f_{s 1} f_{s 2} f_{s 3}}\right) \\
& +\left[\left[\frac{\sqrt{2\left(f_{w s 3 s 1}^{2}+f_{w s 2 s 1}^{2}\right)} \sigma_{\phi_{o b s}}}{2 \pi f_{w s 3 s 1} f_{w s 2 s}}\right]\right] .
\end{aligned}
$$

In order to resolve the cycle ambiguity $N_{s 3}-N_{s 1}$, the conditions of the TEC $D$ and the phase noise $\sigma_{\phi_{o b s}}$ for the third and fourth terms on the right side of Eq. (7) can be derived in the same way.

$$
\begin{gathered}
|D|<\left|\frac{f_{s 1} f_{s 2} f_{s 3}}{2 k f_{w s 3 s 1}\left(f_{s 2}-f_{s 3}\right)}\right| . \\
\sigma_{\phi_{o b s}}<\frac{\pi f_{w s 2 s 1}}{\sqrt{2\left(f_{w s 3 s 1}^{2}+f_{w s 2 s}^{2}\right)}} .
\end{gathered}
$$

Thirdly, the cycle ambiguity of the carrier wave $s 1$ is resolved. By eliminating $\tau_{r e s, s}$ from Eqs. (1) and (6), the following equation is obtained.

$$
\begin{aligned}
& \frac{\phi_{o b s, s 1}}{2 \pi f_{s 1}}-\frac{\phi_{o b s, w s 3 s 1}}{2 \pi f_{w s 3 s 1}} \\
& =\frac{N_{s 1}}{f_{s 1}}-\frac{N_{s 3}-N_{s 1}}{f_{w s 3 s 1}}-k D\left(\frac{1}{f_{s 1}^{2}}+\frac{1}{f_{s 3} f_{s 1}}\right) \\
& +\left[\left[\frac{\sqrt{2 f_{s 1}^{2}+f_{w s 3 s 1}^{2}} \sigma_{\phi_{o b s}}}{2 \pi f_{s 1} f_{w s 3 s 1}}\right]\right] .
\end{aligned}
$$

In order to resolve the cycle ambiguity $N_{s 1}$, the conditions of the TEC $D$ and the phase noise $\sigma_{\phi_{o b s}}$ for the third and fourth terms on the right side of Eq. (10) can be derived in the same way.

$$
\begin{gathered}
|D|<\frac{f_{s 1} f_{s 3}}{2 k\left(f_{s 3}+f_{s 1}\right)} . \\
\sigma_{\phi_{o b s}}<\frac{\pi f_{w s 3 s 1}}{\sqrt{2 f_{s 1}^{2}+f_{w s 3 s}^{2}}} .
\end{gathered}
$$

Finally, the cycle ambiguity of the carrier wave $x$ will be resolved. The delay difference $\delta \tau_{\text {res }}$ is introduced as $\delta \tau_{\text {res }}=$ $\tau_{r e s, x}-\tau_{r e s, s}$. In order to eliminate the $\tau_{r e s, s}$ in Eq. (1), the following equation is formed as follows,

$$
\begin{aligned}
& \frac{\phi_{o b s, x}}{2 \pi f_{x}}-\frac{\phi_{o b s, s 1}}{2 \pi f_{s 1}}=\delta \tau_{r e s}+\frac{N_{x}}{f_{x}}-\frac{N_{s 1}}{f_{s 1}} \\
& -k D\left[\frac{1}{f_{x}^{2}}-\frac{1}{f_{s 1}^{2}}\right]+\left[\left[\frac{\sqrt{\left(f_{s 1}^{2}+f_{x}^{2}\right)} \sigma_{\phi_{o b s}}}{2 \pi f_{s 1} f_{x}}\right]\right] .
\end{aligned}
$$

In order to resolve the cycle ambiguity $N_{x}$, the conditions of the TEC $D$ and the phase noise $\sigma_{\phi_{o b s}}$ for the fourth and fifth terms on the right side of Eq. (13) can be derived in the same 
way.

$$
\begin{aligned}
|D|<\left|\frac{f_{s 1}^{2} f_{x}}{2 k\left(f_{s 1}^{2}-f_{x}^{2}\right)}\right| & . \\
\sigma_{\phi_{o b s}}< & \frac{\pi f_{s 1}}{\sqrt{\left(f_{s 1}^{2}+f_{x}^{2}\right)}} .
\end{aligned}
$$

\subsection{Conditions about the phase noise, the accuracy of} the delay prediction and TEC

Four conditions have been obtained about the phase noise of the received signal in Eqs. (5), (9), (12) and (15). In order to resolve all ambiguity, the condition about the phase noise should be less than the minimum one of them. If the frequencies are $f_{s_{1}}=2212 \mathrm{MHz}, f_{s_{2}}=2218 \mathrm{MHz}$, $f_{s_{3}}=2287 \mathrm{MHz}$ and $f_{x}=8456 \mathrm{MHz}$, the minimum value for the condition will be obtained as follows

$$
\sigma_{\phi_{o b s}}<4.3 \mathrm{deg} .
$$

which comes from Eq. (12). This condition should be considered in realization of MFV.

The condition of the prediction accuracy of the geometric delay is given by Eq. (3) as follows,

$$
\tau_{\text {res }, s}<8.3 \times 10^{-8} \mathrm{sec} .
$$

The result corresponds to $4.8 \mathrm{~km}$ of position accuracy around the Moon for $2000 \mathrm{~km}$ baseline length. This condition can be easily satisfied by using current positioning techniques such as range and range rate measurements.

The four conditions of the ionospheric fluctuation in Eqs. (4), (8), (11) and (14) are also obtained. The most severe one is shown in Eq. (14) and the result follows in the same way,

$$
D<2.3 \times 10^{15} \text { electrons } \mathrm{m}^{-2} \text {. }
$$

This condition can be satisfied easily, considering the results of the daily TEC variations estimated from the global GPS surveying (Tsuchiya and Tsuji, 1995).

\subsection{TEC estimation error}

TEC can be estimated by using the phases of $\mathrm{s} 1$ and $\mathrm{x}$ carrier wave as follows,

$$
\begin{aligned}
D= & -\frac{f_{s 1}^{2} f_{x}^{2}}{k\left[f_{x}^{2}-f_{s 1}^{2}\right]}\left(\frac{\phi_{o b s, s 1}}{2 \pi f_{s 1}}-\frac{\phi_{o b s, x}}{2 \pi f_{x}}-\frac{N_{s 1}}{f_{s 1}}+\frac{N_{x}}{f_{x}}\right) \\
& -\frac{f_{s 1}^{2} f_{x}^{2} \delta \tau_{r e s}}{k\left[f_{x}^{2}-f_{s 1}^{2}\right]}+\frac{f_{s 1} f_{x} \sqrt{f_{s 1}^{2}+f_{x}^{2}}}{2 \pi k\left[f_{x}^{2}-f_{s 1}^{2}\right]}\left[\left[\sigma_{\phi_{o b s}}\right]\right] .
\end{aligned}
$$

The second term in the right side of Eq. (19) means the effect of the displacement between the antennas of S-band and Xband. Assuming that the displacement of the antennas is $20 \mathrm{~cm}$ in the case of the relay satellite of SELENE, $\delta \tau_{\text {res }}$ is $3.5 \times 10^{-12} \mathrm{sec}$. in delay difference for $2000 \mathrm{~km}$ baseline. The second term $\sigma_{D_{\tau_{S X}}}$ is calculated as,

$$
\sigma_{D_{\tau_{S X}}}=1.4 \times 10^{14} \text { electrons } \mathrm{m}^{-2} .
$$

The third term in Eq. (19) means the effect of the phase noise $\sigma_{\phi_{o b s}}$ on the estimation of the TEC. Substitution the value of $\sigma_{\phi_{o b s}}$ in Eq. (16), value of the third term $\sigma_{D_{o b s}}$ in Eq. (19) is caluculated as,

$$
\sigma_{D_{o b s}}=2.2 \times 10^{14} \text { electrons } \mathrm{m}^{-2} .
$$

Comparing Eqs. (20) with (21), the effect of the displacement between the two antennas which is expected in SELENE relay satellites is not dominant in the estimation of the TEC. However, the displacement between the antennas of S-band and X-band is important at designing the MFV mission.

\subsection{Geometric delay}

The geometric delay $\tau_{r e s, x}$ is obtained from Eq. (1) as follows,

$$
\tau_{r e s, x}=\frac{\phi_{o b s, x}-2 \pi N_{x}}{2 \pi f_{x}}+k D \frac{1}{f_{x}^{2}}+\frac{\left[\left[\sigma_{\phi_{o b s}}\right]\right]}{2 \pi f_{x}} .
$$

The estimation errors $\sigma_{D_{\tau_{S X}}}$ in Eq. (20) and $\sigma_{D_{o b s}}$ in Eq. (21) of the TEC result in the geometric delay estimation error $\sigma_{\tau_{\sigma_{D}}}$ as follows,

$$
\sigma_{\tau_{\sigma_{D}}}=3.4 \times 10^{-13} \mathrm{sec} .
$$

This error corresponds to a small position error of $1.5 \mathrm{~cm}$ around the Moon for the $2000 \mathrm{~km}$ baseline.

Substituting Eq. (16) into the third term, the effect of the phase noise error on the estimation of the geometric delay is obtained as,

$$
\sigma_{\tau}=\frac{\left[\left[\sigma_{\phi_{o b s}}\right]\right]}{2 \pi f_{x}}=1.4 \times 10^{-12} \mathrm{sec} .
$$

This error corresponds to a small position error of $8 \mathrm{~cm}$ around the Moon for the $2000 \mathrm{~km}$ baseline.

\subsection{Effects on the residual fringe phase by frequency variations}

The residual fringe phase depends on the frequency of the transmitted radio signal, and the variation of it affects the residual fringe phase. In order to know the phase error by the frequency variation, we introduce $f_{i}^{\prime}$ instead of $f_{i}$ as follows,

$$
f_{i}^{\prime}=f_{i}+\delta f_{i} \text {. }
$$

Substituting $f_{i}^{\prime}$ into the first and third terms on the right side of Eq. (1), the effect on the phase by the frequency variation can be less than the limit shown in Eq. (16) as follows,

$$
\begin{gathered}
\left|2 \pi\left(f_{i}+\delta f_{i}\right) \tau_{r e s, j}-2 \pi f_{i} \tau_{r e s, j}\right|=\left|2 \pi \delta f_{i} \tau_{r e s, j}\right|<\sigma_{\phi_{o b s}}, \\
\left|2 \pi k D \frac{1}{f_{i}}-2 \pi k D \frac{1}{f_{i}+\delta f_{i}}\right|=\left|\frac{2 \pi k D \delta f_{i}}{f_{i}\left(f_{i}+\delta f_{i}\right)}\right|<\sigma_{\phi_{o b s}} .
\end{gathered}
$$

By using the values in Eqs. (16), (17) and (18), the condition about the frequency variation should be least one of them as follows,

$$
\delta f_{i}<140 \mathrm{kHz}
$$

In general, a frequency variation $\delta f_{i}$ can be roughly expressed with normalized frequency stability $\sigma_{y}(\tau)$ as, $\delta f_{i}=$ $\sigma_{y}(\tau) f_{i}$, where $\tau$ is a integration duration and $f_{i}$ is the frequency. Finally the condition of frequency variations can be roughly obtained as follows,

$$
\sigma_{y}(\tau)<1.7 \times 10^{-5}
$$

This condition about the frequency variation is also a key condition for MFV. 


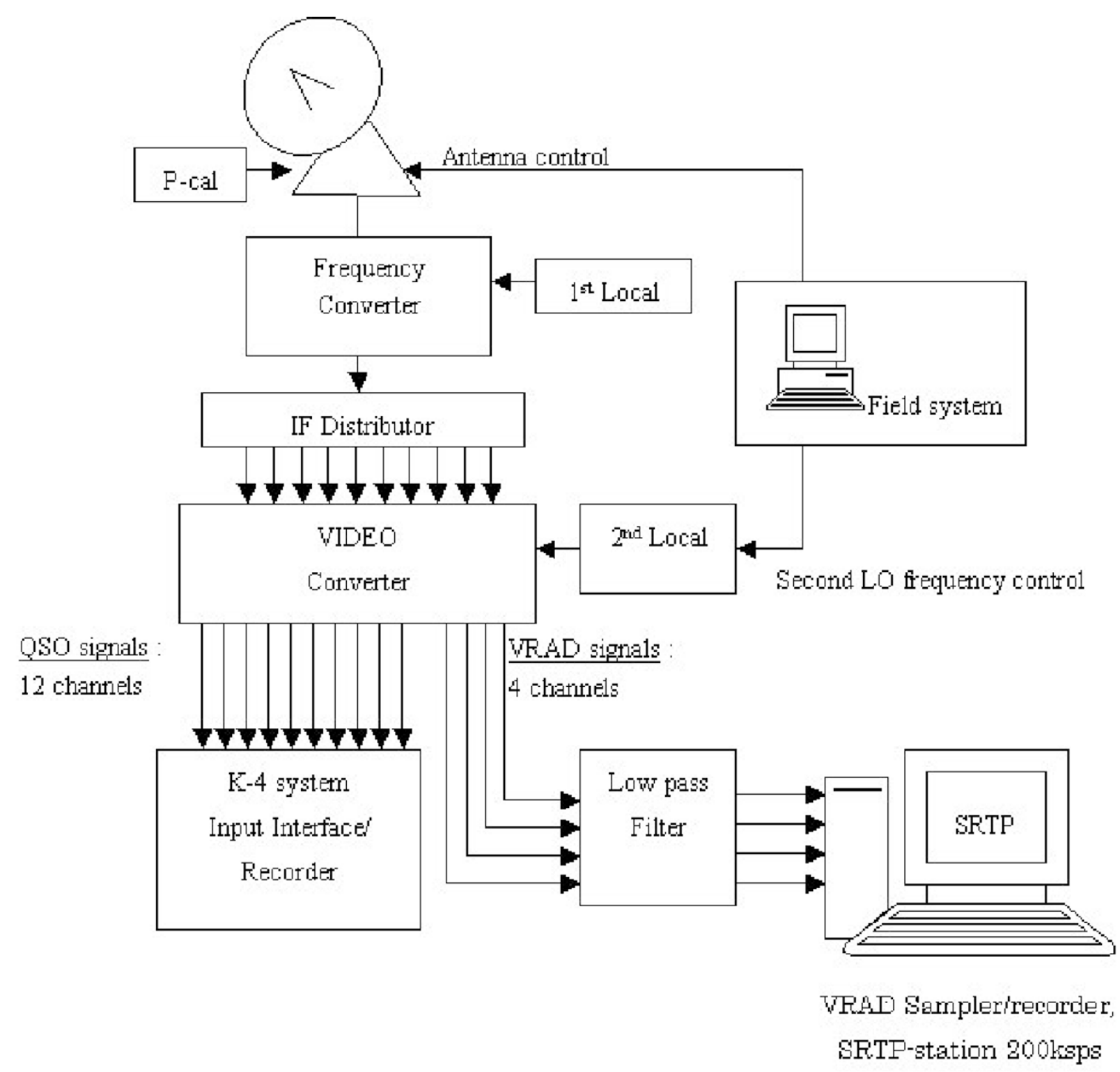

Fig. 2. Block diagram of the observation system.

\section{Application of MFV}

MFV method will be realized under the Japanese lunar exploring program, SELENE. The radio sources will be installed in the relay satellite and VRAD satellite (Iwata et $a l ., 2001)$. The transmitting power of the radio sources and the ground system for MFV are summarized in this section. 3.1 Radio sources for MFV

Transmitting power of radio sources for MFV measurements is estimated from the relation between the fringe phase error $\sigma_{\phi}$ for each radio source and the flux density of the sources $S_{c}\left(\mathrm{Wm}^{-2} \mathrm{~Hz}^{-1}\right)$ as

$$
\sigma_{\phi}=\frac{8 k}{\pi S_{c} D_{1} D_{2} h} \sqrt{\frac{B T_{1} T_{2}}{2(\Delta v)^{2} t \eta_{1} \eta_{2}}},
$$

where $k$ is Boltzmann's constant $\left(k=1.38 \times 10^{-23} \mathrm{JK}^{-1}\right)$, $B$ is recording bandwidth $(\mathrm{Hz}), T_{i}$ is system temperature at each station $(\mathrm{K}), D_{i}$ is diameter of each antenna $(\mathrm{m})$, $h$ is a coherence factor, $\Delta v$ is bandwidth of the signal
$(\mathrm{Hz}), t$ is integration time (sec.), and $\eta_{i}$ is aperture efficiency of each antenna. The source flux density of $S_{c}=$ $1.2 \times 10^{-23} \mathrm{Wm}^{-2} \mathrm{~Hz}^{-1}$ (1200 Jy) is necessary for $\sigma_{\phi}=0.05$ (4.3/ $\sqrt{2}$ degrees) in X-band with $B=140 \mathrm{~Hz}, T_{i}=380 \mathrm{~K}$, $D_{i}=20 \mathrm{~m}, h=0.95, \Delta v=B, t=100 \mathrm{~s}$, and $\eta_{i}=0.35$. The values of the parameters are based on VERA antenna of 20 meter in diameter of National Astronomical Observatory, Mizusawa (Honma et al., 2000). The system temperature includes the contribution from the Moon of $200 \mathrm{~K}$ which is the surface temperature of the Moon at noon. The total flux on the Earth becomes $F=S_{c} \times \Delta v=1.7 \times 10^{-21} \mathrm{Wm}^{-2}$, which corresponds to the equivalent isotropic radiated power (e.i.r.p) of $P=4 \pi F l^{2}=38 \mathrm{~mW}$ with the distance between the Earth and the Moon of $l=3.8 \times 10^{8} \mathrm{~m}$, taking an atmospheric absorption loss and a rain loss which amount to $-5.8 \mathrm{~dB}$ in all and the link margin of $5.1 \mathrm{~dB}$ into consideration. The radiated power of the carrier wave in X-band required for the transmitter becomes $60 \mathrm{~mW}$, considering the feed loss of $-2 \mathrm{~dB}$. 


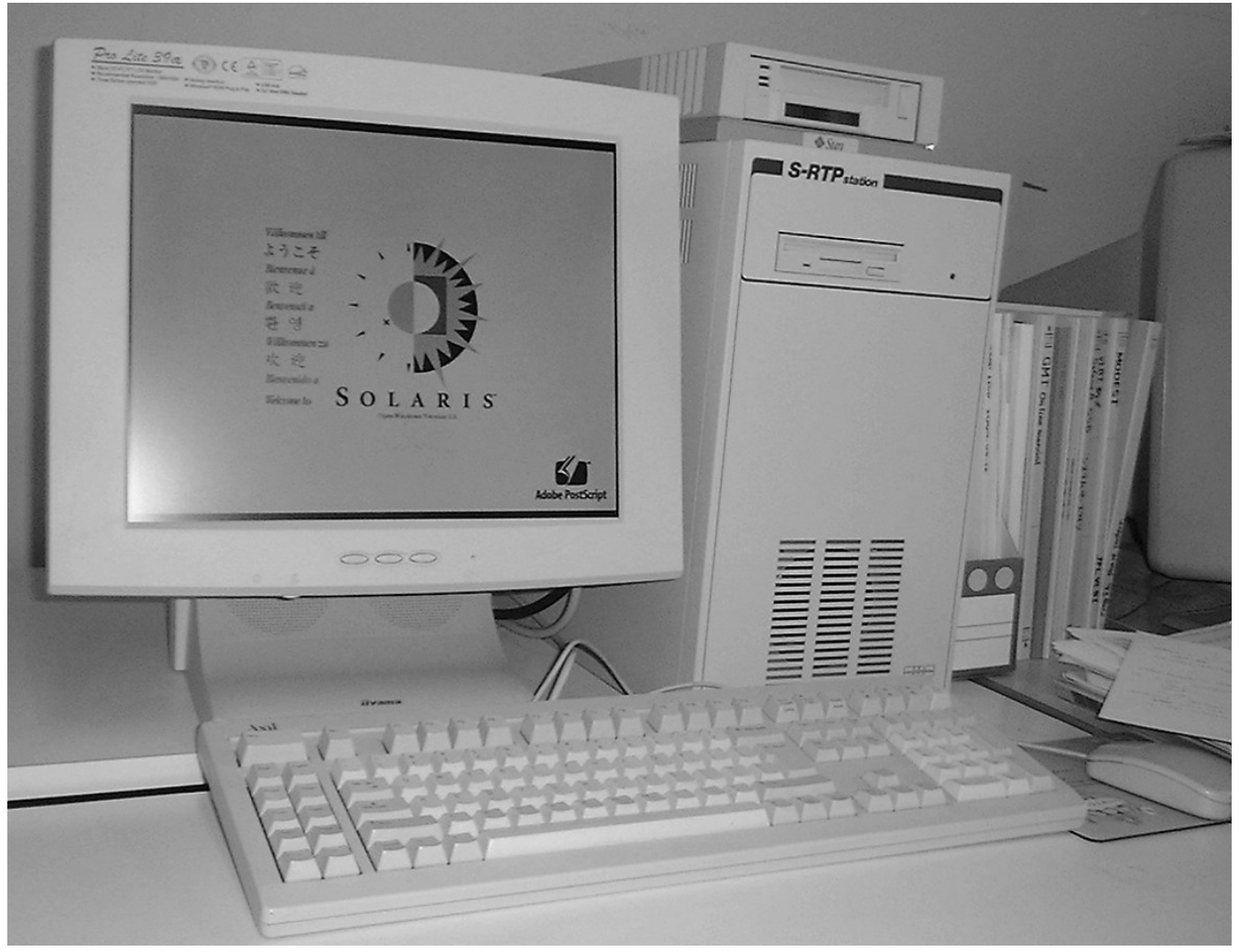

Fig. 3. Narrow bandwidth sampler for MFV.

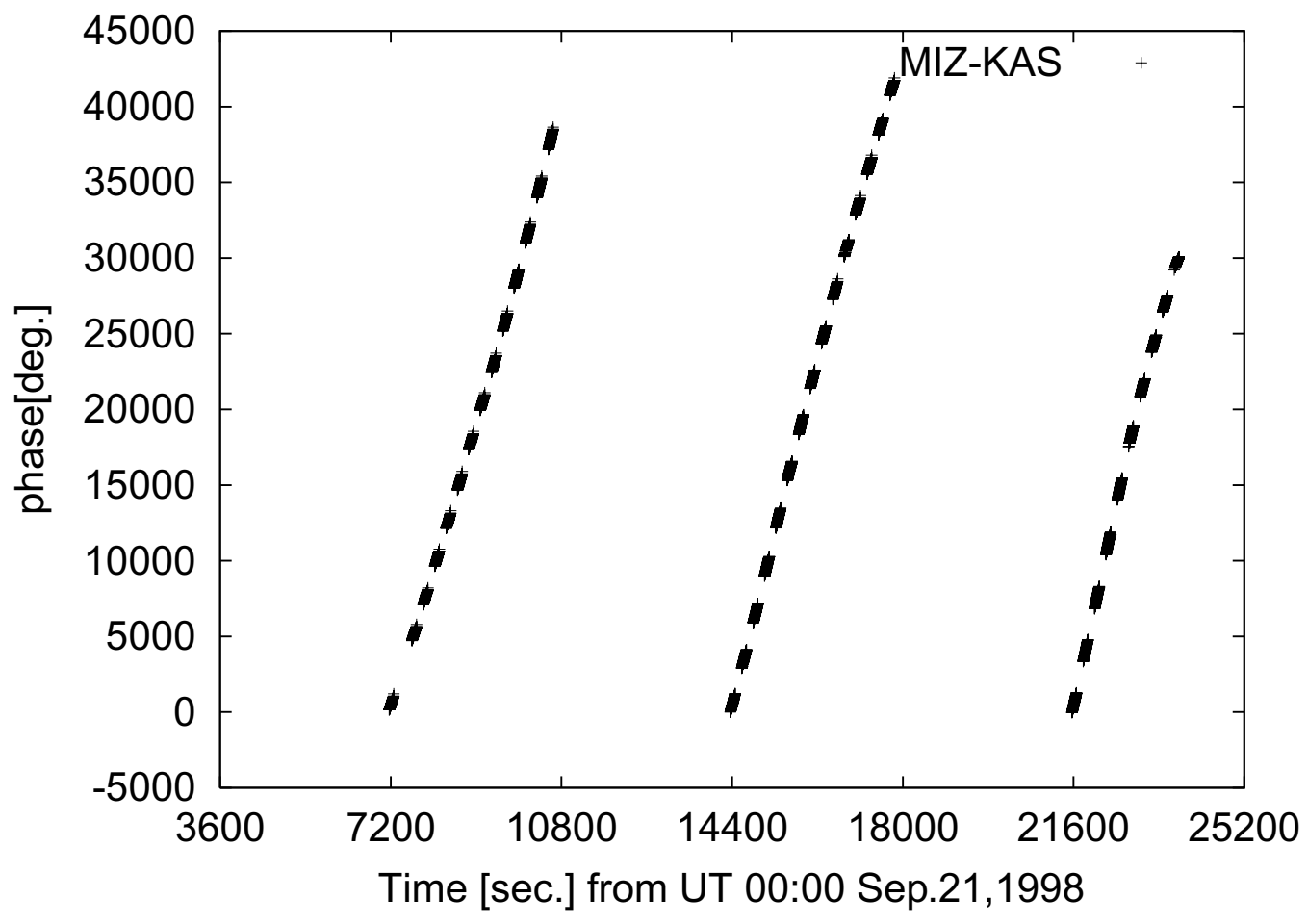

Fig. 4. Residual fringe phases of Lunar Prosepector. 


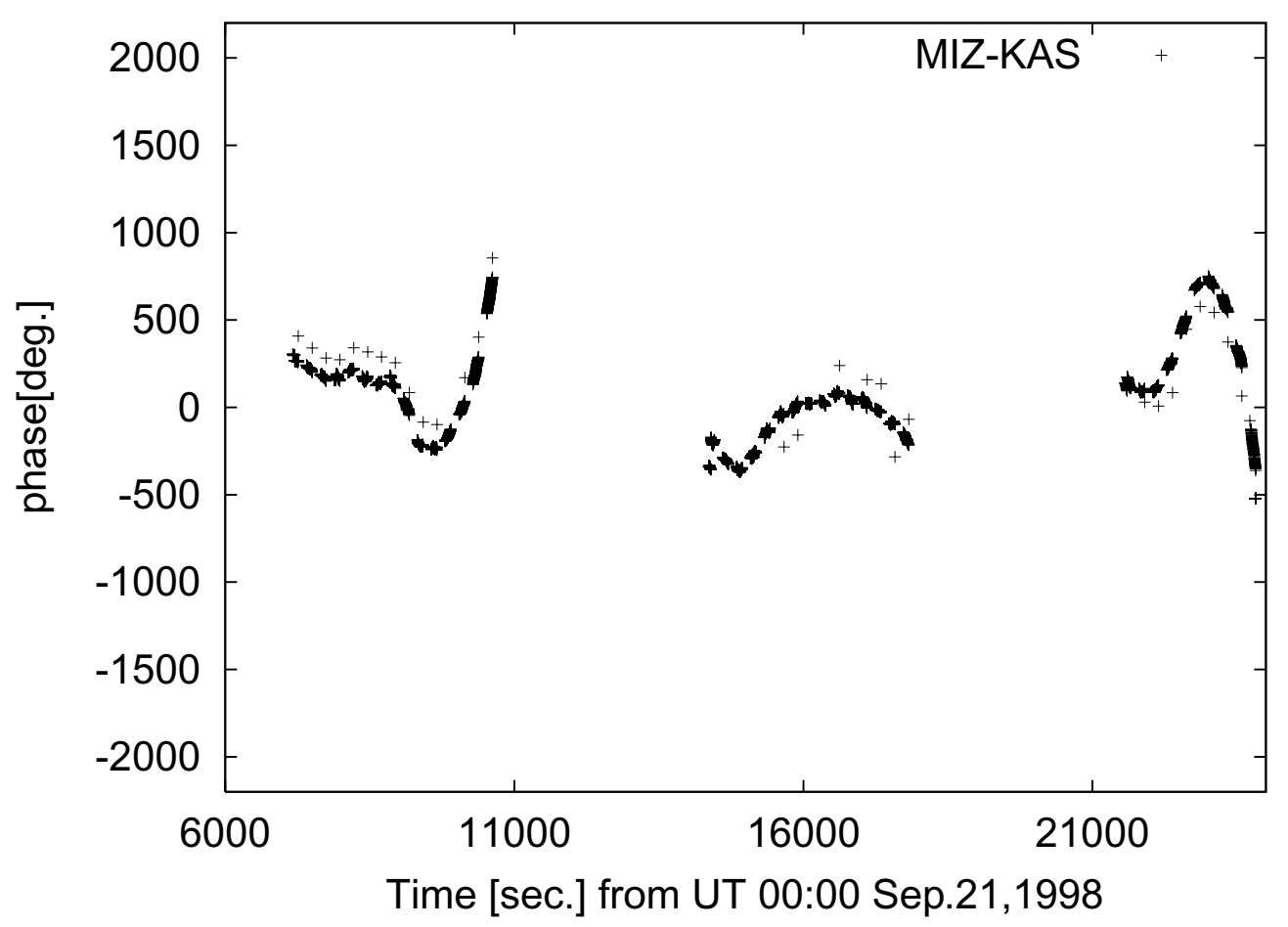

Fig. 5. The residual fringe phase of Lunar Prosepector. The linear component and ionospheric effect are removed. The initial orbital element $\Omega$ is offset by +0.08 degrees.

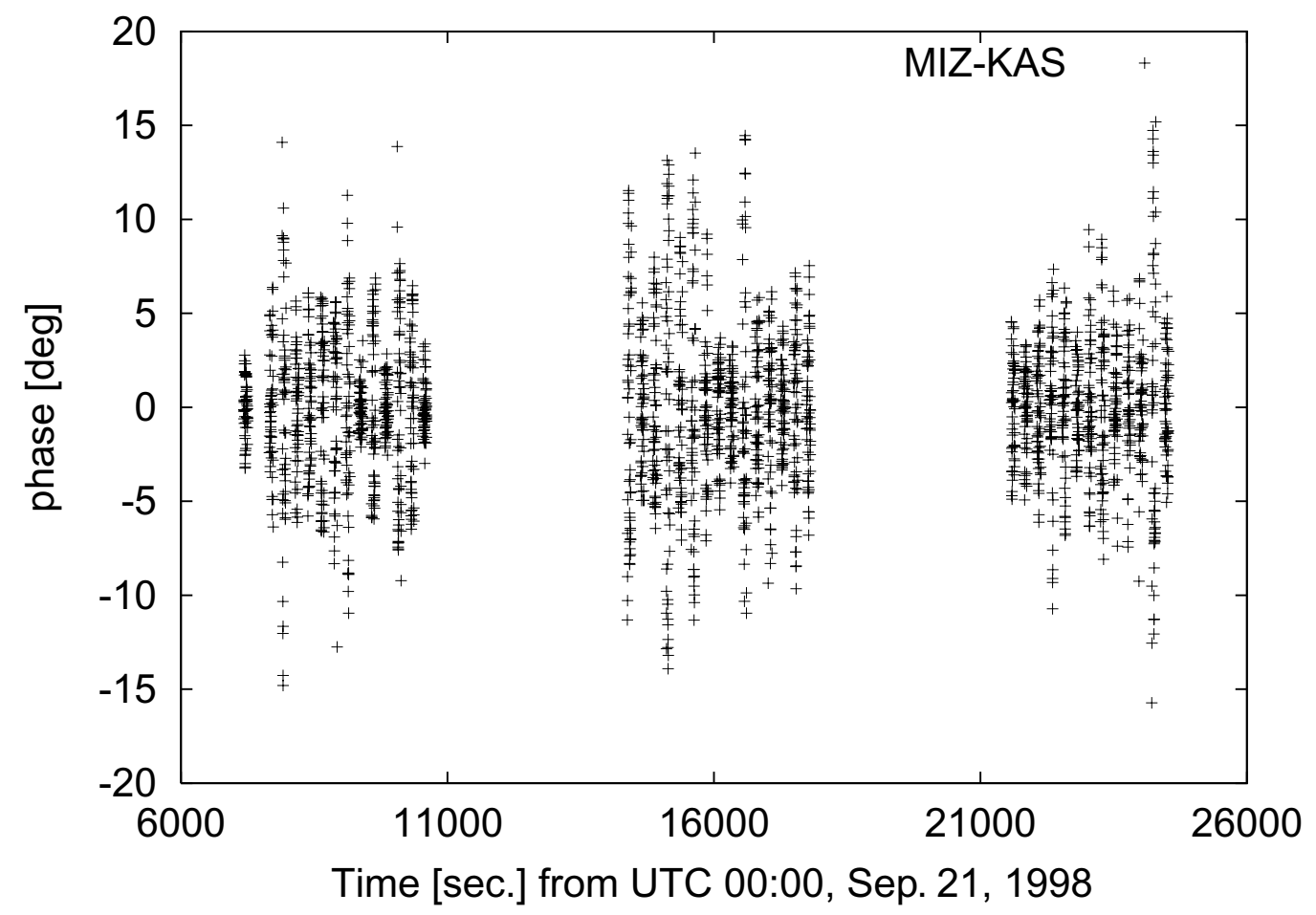

Fig. 6. The short period variation of the residual fringe phase. 
The radiated power per carrier wave at S-band, on the other hand, becomes $100 \mathrm{~mW}$ when estimating by using the same parameters as X-band except $\eta_{i}=0.14$ and $T_{i}=267$ $\mathrm{K}$ which includes the contribution from the Moon of $94 \mathrm{~K}$ with a half power beam width of 0.4 degrees. These link designs are minutely described by Hanada et al. (1999).

\subsection{Ground system for MFV}

A new ground VLBI system, which consists of hardware to sample and record the carrier waves and software to estimate phase delays, is developed. The block diagram is shown in Fig. 2. The hardware samples and digitizes the video signals at sampling rate of $200 \mathrm{kHz}$ and records them on an $8 \mathrm{~mm}$ tape. The narrow bandwidth sampler and recorder is shown in Fig. 3. The new software crosscorrelates the recorded signals and produces the phase delays. The product is inputted to another software to estimate orbits and gravity fields (Matumoto et al., 2002).

\section{Preliminary Experiment of MFV by using Lu- nar Prospector}

As a preliminary test of the whole system, an experiment of Lunar Prospector was carried out in Sep. 21, 1998. The antennas involved in the observation were the Kashima 34 m-diameter antenna of Communication Research Laboratory and the Mizusawa $10 \mathrm{~m}$-diameter antenna of National Astronomical Observatory. The developed ground system was used to record and estimate the fringe phases. Unfortunately Lunar Prospector transmitted only one carrier wave, so that the test for resolving the ambiguity of the fringe phase could not be achieved. However, phase error for the carrier wave and the effect of TEC can be evaluated by using Lunar Prospector data.

The integration period is set at about 1.3 seconds which corresponds to 262,144 time-series data. Although interval of antenna switching is 120 seconds, successive data for only about 90 seconds are processed eliminating data for antenna slew time loss of about 30 seconds. The switching interval time is selected considering the minimum switching interval limited by the old field system installed at one of the ground stations. The residual fringe phases from 01:00 to 07:00 on Sep. 21, 1998 in UT are shown in Fig. 4. A series of the successive residual fringe phases for 90 seconds could be connected continuously for about one hour, a half period of Lunar Prospector's orbit, by the following way. The residual fringe phases for the first period of 90 seconds are obtained with the cycle ambiguity, and then its derivative in time is calculated. The derivative enabled us to predict the next successive residual fringe phases 240 seconds later. The residual fringe phase in the second period, therefore, could be determined nearest the predicted phase. The third residual fringe phase could be also determined from the derivative of the residual fringe phase in the second period in the same way, and finally a series of successive residual fringe phases were determined without ambiguity of $2 \pi$ over about one hour of the path. Although a series of residual fringe phases were obtained, we could not estimate the cycle ambiguity of the residual fringe phases in the first period every one hour of the path because Lunar Prospector emitted only one carrier wave. If Lunar Prospector had transmitted four carrier waves of appropriate frequencies, the cycle ambiguity could be resolved, and the phase delay could be obtained without ambiguity of $2 \pi$ for all the period.

'The linear trend in Fig. 4 corresponds to $13 \mathrm{ps} / \mathrm{s}$ in delay rate. This seems to be the predicted delay model error in the correlation process and the frequency difference between frequency standards at the two VLBI stations. Subtracting the linear component from the trend, the residuals are obtained. There, however, remains a variation of fringe phases with a period around a few thousand seconds. The residual fringe phases after the correction for the ionospheric delay, which is calculated by the TEC global model provided by The Center for Orbit Determination in Europe (CODE), University of Berne, Switzerland, have systematic variations of about 1000 degrees with period of about one hour. These variations are supposed to be caused by the errors in the initial orbital elements and model errors of the lunar gravity fields which are used for the prediction of the geometric delay. In order to investigate effects of the initial orbit error, we calculate the residual fringe phase by adding some small offset to each initial orbital element. This processing makes it possible to roughly judge if there are errors in the initial orbital elements. As the results, the variation is minimized for the orbital element of Longitude of ascending node $\Omega+0.08$ degrees (Fig. 5). Although these long period variations can not be estimated in this experiment, they will be estimated in SELENE mission by MFV. The short period variations of the residual fringe phases will remain even if the two variations are estimated. The RMS of the postfit residual from the 4th order polynomials for the period of several tens seconds is about 4.4 degrees (Fig. 6). The RMS residual for several seconds means that we can determine the position of Lunar Prospector within the error of $1.5 \mathrm{~m}$ around the Moon with this $350 \mathrm{~km}$ Baseline if we can correct the variations with long period by using the MFV.

\subsection{Conclusion}

MFV method, which enables us to obtain the phase delay of RF signals at $\mathrm{S}$ and $\mathrm{X}$-band without cycle ambiguity, is proposed. The several conditions of the realization of this method are summarized. The new ground VLBI system, which consists of hardware to sample and record the received signals and software to estimate the phase delay, has been developed.

The preliminary experiment was carried out by using lunar orbiter, Lunar Prospector. Unfortunately Lunar Prospector transmitted only one carrier wave, so that the test for resolving the ambiguity of the fringe phase could not be achieved. The residual fringe phases after the correction for the ionospheric delay have systematic variations of about 1000 degrees with period of about one hour. These variations are supposed to be caused by the errors in the initial orbital elements and model errors of the lunar gravity fields used. The RMS of the residual fringe phases averaged for several ten seconds reaches about 4 degrees, which corresponds to 1.5 $\mathrm{m}$ in the positioning error around the Moon. It is confirmed that the hardware and software system have enough availability to achieve the expected accuracy in MFV.

Acknowledgments. We would like to thank all the staffs of Kashima space communication research center of CRL, GSI and Mizusawa observation center of NAO for their helps in operating the VLBI network. 


\section{References}

Border, J. S., W. M. Folkner, R. D. Kahn, and K. S. Zukor, Precise Tracking of the Magellan and Pioneer Venus Orbiters by Same-Beam Interferometry, Part I: Data Accuracy Analysis, TDA progress report, 42-110, Aug. $15,1992$.

Hanada, H., T. Iwata, N. Kawano, K. Heki, S. Tsuruta, T. Ishikawa, H. Araki, K. Matsumoto, Y. Kono, Y. Kaneko, M. Ogawa, Y. Iijima, Y. Koyama, M. Hosokawa, T. Miyazaki, N. Namiki, A. Sengoku, Y. Fukuzaki, T. Ikeda, F. Fuke, and RISE group, Observation system of radio sources on the Moon by VLBI in SELENE project, Proceedings of the International Workshop on GEMSTONE, Jun., 25-28, 126-130, 1999.

Hatch, R. R., The synergism of GPS code and carrier measurements, Proc. 3rd Int. Symp. on Satellite Doppler Positioning, New Mexico, 8-12, Feb., 1213-1231, 1982.

Honma, M., T. Oyama, K. Hachisuka, S. Sawada-Satoh, K. Sebata, M. Miyoshi, O. Kameya, S. Manabe, N. Kawaguchi, T. Sasao, S. Kameno, K. Fujisawa, K. Shibata, T. Bushimata, T. Miyaji, H. Kobayashi, M. Inoue, H. Imai, H. Araki, H. Hanada, K. Iwadate, Y. Kaneko, S. Kuji, K. Sato, S. Tsuruta, S. Sakai, Y. Tamura, K. Horiai, T. Hara, K. Yokoyama, J. Nakajima, E. Kawai, H. Okubo, H. Osaki, Y. Koyama, M. Sekido, T. Suzuyama, R. Ichikawa, T. Kondo, K. Sakai, K. Wada, N. Harada, N. Tougou, M. Fujishita, R. Shimizu, S. Kawaguchi, A. Yoshimura, M. Nakamura, W. Hasegawa, S. Morisaki, R. Kamohara, T. Funaki, N. Yamashita, T. Watanabe, T. Shimoikura, M. Nishio, T. Omodaka, and A. Okudaira, J-Net Galactic Plane Survey of VLBI Radio Sources for VLBI
Exploration of Radio Astrometry (VERA), Publ. Astron. Soc. Japan, 52, 631-643, 2000.

Iwata, T., M. Takahashi, N. Namiki, H. Hanada, N. Kawano, K. Heki, K. Matsumoto and T. Takano, Mission instruments for lunar gravity measurements using SELENE sub-satellites, Journal of the Geodetic Society of Japan , 47, 1, 558-563, 2001.

Matumoto, K, K. Heki, and H. Hanada, Global lunar gravity field recovery from SELENE, IVS 2002 General Meeting Proceedings, edited by N. R. Vandenberg and K. D. Baver, NASA/CP-2002-210002.

Sagdeyev, R. Z., V. V. Kerzhanovitch, L. R. Kogan, V. I. Kostenko, V. M. Linkin, L. I. Matveyenko, R. R. Nazirov, S. V. Pogrebenko, I. A. Struckov, R. A. Preston, J. Purcel, C. E. Hildebrand, V. A. Grishmanovskiy, A. N. Kozlov, E. P. Molotov, J. E. Blamont, L. Boloh, G. Laurans, P. Kaufmann, J. Galt, F. Biraud, A. Boischot, A. Ortega-Molina, C. Rosolen, G. Petit, P. G. Mezger, R. Schwartz, B. O. Ronnang, R. E. Spencer, G. Nicolson, A. E. E. Rogers, M. H. Cohen, R. M. Martiroysan, I. G. Moiseyev, and J. S. Jatskiv, Differential VLBI measurements of the Venus atmosphere dynamics by balloons: VEGA project, Astron. and Astrophys., 254, 387392, 1992.

Tsuchiya, A. and H. Tsuji, GPS Sokuryo-no Kiso, Japan Surveying Association, 11-170, 1995 (in Japanese).

Y. Kono (e-mail: kono.yusuke@nao.ac.jp) 